www.nature.com/jhg

\title{
Single human papillomavirus 16 or 52 infection and later cytological findings in Japanese women with NILM or ASC-US
}

\author{
Shuhei Abe ${ }^{1}$, Kiyonori Miura ${ }^{1}$, Akira Kinoshita $^{2}$, Hiroyuki Mishima ${ }^{2}$, Shoko Miura ${ }^{1}$, Kentaro Yamasaki ${ }^{1}$, \\ Yuri Hasegawa ${ }^{1}$, Ai Higashijima ${ }^{1}$, Ozora Jo $^{1}$, Atsushi Yoshida ${ }^{1}$, Masanori Kaneuchi ${ }^{1}$, Koh-ichiro Yoshiura ${ }^{2}$ \\ and Hideaki Masuzaki ${ }^{1}$
}

The relationship between oncogenic human papillomavirus (HPV) infection and later cytological findings in the uterine cervix is unknown in women who were negative for intraepithelial lesion and malignancy (NILM) or atypical squamous cells of undetermined significance (ASC-US). This was investigated in this study in a Japanese population to determine the clinical utility of oncogenic (HPV) genotyping. The relative risk of progressive cytological findings 2 years after identification of oncogenic HPV infection was higher than in cases of non-oncogenic HPV infection (relative risk $3.827 ; 95 \%$ confidence interval (CI): 1.282-11.422), as well as in cases of negative HPV infection (relative risk 2.124; 95\% Cl: 1.451-3.110). Moreover, the relative risk of progression of cytological findings 2 years later in cases of HPV-16 infection was higher than in cases of HPV-52 infection (relative risk 2.094; 95\% Cl: 1.005-3.935). Therefore, the initial HPV-DNA genotype may be a potential predictive marker of later progression of cytological findings in the uterine cervix in cases of NILM or ASC-US.

Journal of Human Genetics (2014) 59, 251-255; doi:10.1038/jhg.2014.9; published online 13 February 2014

Keywords: cytological findings; genotype; human papillomavirus; oncogenic HPV-16; oncogenic HPV-52; uterine cervical cancer

\section{INTRODUCTION}

Persistent infections with oncogenic human papillomaviruses (HPVs), including 16 different HPV genotypes (16, 18, 31, 33, 35, 45, 51, 52, $53,56,58,59,66,68,73$ and 82 ), are recognized as a major risk factor for the development of cervical cancer. ${ }^{1}$ HPV-16 and HPV-18 infection accounts for approximately $70 \%$ of cancers and $50 \%$ of high-grade cervical intraepithelial neoplasia. ${ }^{2}$ Although the risk of cervical cancer may be an order of magnitude higher for HPV-16 infection than other oncogenic HPV types, our previous studies showed that HPV-52 was a more common genotype in Nagasaki, Japan, compared with the distribution of high-risk HPV genotypes in other countries. ${ }^{3,4}$ In addition, HPV-52 was the most common genotype among HPV-infected pregnant Japanese women. The second most common genotype was HPV-16, and these two genotypes collectively accounted for around 60\% of HPV-positive pregnant women. ${ }^{4}$ Hence, geographic variations in HPV-type distributions should be an important consideration.

The screening of high-grade squamous intraepithelial lesions (HSILs) and cervical cancer currently involves the detection of oncogenic HPV by cervicovaginal Pap smears, ${ }^{5}$ and it is both important and necessary to clarify the association between the individual oncogenic HPV genotypes in each region and the progression of later cytopathological findings in the uterine cervix. When women are screened using cervicovaginal Pap smears, most are diagnosed with negative for intraepithelial lesion or malignancy (NILM) or atypical squamous cells of undetermined significance (ASC-US). ASC-US consists of various cytological abnormalities, which may contain low-grade squamous intraepithelial lesions or HSILs of the uterine cervix. Therefore, testing for oncogenic HPV is now routinely carried out in both Japan and the United States for managing women with ASC-US. However, the clinical usefulness of oncogenic HPV testing is limited by the fact that oncogenic HPV infections are relatively common among women without cervical intraepithelial neoplasia (CIN) or cancer. Also, in the United States, the clinical usefulness of HPV-16 and/or HPV-18 genotyping to triage oncogenic HPV-positive women with NILM aged 30 years and above has been recently recognized in clinical management guidelines; HPV16-positive or HPV-18-positive women with NILM are referred for colposcopy, whereas other oncogenic HPV-positive women with NILM are recommended to repeat the cervical cytology and oncogenic HPV testing in 12 months. ${ }^{6}$ Nevertheless, the data supporting the use of HPV genotyping in this manner are relatively

${ }^{1}$ Department of Obstetrics and Gynecology, Nagasaki University Graduate School of Biomedical Sciences, Nagasaki, Japan and ${ }^{2}$ Department of Human Genetics, Nagasaki University Graduate School of Biomedical Sciences, Nagasaki, Japan

Correspondence: Dr K Miura, Department of Obstetrics and Gynecology, Nagasaki University Graduate School of Biomedical Sciences, 1-7-1 Sakamoto, Nagasaki 852-8501, Japan.

E-mail: kiyonori@nagasaki-u.ac.jp

Received 11 October 2013; revised 17 January 2014; accepted 17 January 2014; published online 13 February 2014 
limited. Moreover in clinical management, it is not obvious how oncogenic HPV-positive women with NILM or ASC-US cytology should be managed in primary cervical cancer screening, or how the presence of oncogenic HPV infection affects later cytological findings in Japanese women with a cytological diagnosis of NILM or ASC-US at first admission.

Therefore, this study aimed to understand the clinical utility of oncogenic HPV genotyping in Japanese women by investigating the relationship between oncogenic HPV infection in a Japanese population and cytological changes in the uterine cervix. Subsequently, we investigated how a single HPV-16 or HPV-52 infection affects the cytological findings in Japanese women with NILM or ASC-US 2 years later.

\section{MATERIALS AND METHODS}

\section{Patients}

Women admitted to five hospitals in Nagasaki Prefecture, Japan, between 2007 and 2011 as a pre-vaccination population for the HPV vaccine ${ }^{3,4}$ were informed of the purpose of the study and gave their consent for participation. We identified 319 cases with a cytological diagnosis of NILM or ASC-US at first admission, and followed them up by cervicovaginal Pap smears and HPV genotyping over the next 2 years. The study protocol was approved by the ethical review board of Nagasaki University and the other hospitals involved.

\section{Sample collection and cytological diagnoses}

Specimens were collected using a Cervex Brush (Rovers Medical Devices, Oss, The Netherlands) and suspended in $10 \mathrm{ml}$ of SurePath preservative fluid (Becton Dickinson, Franklin Lakes, NJ, USA). Samples from the same vial were used for cytological testing with the Bethesda III system (2001) and for HPV genotype testing. ${ }^{3,4}$ Cervical specimens for cytology and HPV genotyping were obtained at each visit from participants who received regular follow-up examinations. To minimize the possibility of diagnostic variations, cytologic diagnoses of the specimens were performed by the same experienced cytoscreener and a qualified medical doctor in a commercial laboratory (SRL, Tokyo, Japan), who were blinded to the results of the HPV genotyping test. Cervical cytological findings were reported as NILM, ASC-US, low-grade squamous intraepithelial lesion, atypical cellscannot exclude HSILs (ASC-H) and HSIL. Regarding cervical cytological findings determined 2 years after first admission, cases showing progression (changing from 'NILM' to 'ASC-US or worse cytological findings', or from 'ASC-US' to 'low-grade squamous intraepithelial lesion or worse cytological findings') were defined as the progression group, and cases showing no change or regression (from 'NILM' to 'NILM', or from 'ASC-US' to 'ASC-US or NILM') were defined as the non-progression group. Colposcopy was performed in the cases diagnosed as ASC-US with oncogenic HPV infections (data not shown).

\section{HPV genotyping test}

Genotyping of HPV DNA in SurePath preservative fluid was carried out after preparing glass slides using the Linear Array HPV Genotyping Test Kit (Roche Molecular Systems, Indianapolis, IN, USA). PGMY09/PGMY11 primers ${ }^{7}$ amplified the L1 conserved region by polymerase chain reaction, and hybridization of the HPV amplicon was performed using an array of oligonucleotide probes that allowed the independent identification of individual HPV genotypes. This kit can detect the following 37 HPV genotypes: $6,11,16,18,26,31,33,35,39,40,42,45,51,52,53,54,55,56,58$, 59, 61, 62, 64, 66, 67, 68, 69, 70, 71, 72, 73 (MM9), 81, 82 (MM4), 83 (MM7), 84 (MM8), IS39 and CP6108 (89). For consistency with previous studies, 16 HPV genotypes $(16,18,31,33,35,45,51,52,53,56,58,59,66,68,73$ and 82) were considered to be high risk as they had previously been related to cervical cancer. ${ }^{1,8,9}$

\section{Statistical analysis}

Patient backgrounds were compared between the progression and nonprogression groups using Student's $t$-tests and $\chi^{2}$ tests for continuous and discrete variables, respectively. Differences in oncogenic HPV genotyping between the two groups were evaluated using relative risk. Statistical analyses were performed with SPSS software version 19 (IBM Japan, Tokyo, Japan). Significant differences were defined as $P<0.05$.

\section{RESULTS}

\section{Study population backgrounds}

Of the 319 Japanese women (253 cases of NILM and 66 cases of ASCUS) originally studied, HPV genotyping showed that 178 (159 cases of NILM and 19 cases of ASC-US) had no HPV infection, 26 (17 cases of NILM and 9 cases of ASC-US) had only a non-oncogenic HPV infection, 38 (25 cases of NILM and 13 cases of ASC-US) had multiple oncogenic HPV infections, and 77 (52 cases of NILM and 25 cases of ASC-US) had a single oncogenic HPV infection (Figure 1). The 77 cases with a single oncogenic HPV infection included HPV-16 ( $n=20 ; 13$ cases of NILM and 7 cases of ASC-US), HPV-18 ( 1 case of NILM), HPV-31 ( $n=7 ; 6$ cases of NILM and 1 case of ASC-US), HPV-35 (2 cases of ASC-US), HPV-39 (1 case of ASC-US), HPV-51 ( $n=3 ; 1$ case of NILM and 2 cases of ASC-US), HPV-52 $(n=29 ; 22$ cases of NILM and 7 cases of ASC-US), HPV-58 $(n=11 ; 6$ cases of NILM and 5 cases of ASC-US), HPV-59 (2 cases of NILM) and HPV68 ( 1 case of NILM) (Figure 1). Of the 25 cases of ASC-US with a single oncogenic HPV infection, 12 were followed up with HPV genotyping. Of the 12 cases of ASC-US with a single oncogenic HPV infection at first admission, persistent infection of oncogenic HPV was detected 2 years later in $4(33.3 \%$; 3 cases showing a cytological 'progression', and 1 case showing a cytological 'nonprogression'), while oncogenic HPV had disappeared in the remaining 8 cases $(66.7 \%$, all 8 cases showing a cytological 'non-progression' 2 years later; $P=0.005, \chi^{2}$ test).

There was no significant difference in patient background between the single oncogenic HPV infection and HPV-negative groups (Table 1), the single oncogenic HPV infection and single nononcogenic HPV infection groups (Table 1), or between the HPV-16 infection and HPV-52 infection groups (Table 2). There was no significant difference in age distributions between the progression and the non-progression groups (Table 3 ).

\section{Relationship between oncogenic HPV infection and the progression of cytological findings in Japanese women with NILM or ASC-US}

Of the 77 cases with a single oncogenic HPV infection, $30(38.96 \%)$ were classified in the progression group, whereas 47 (61.04\%) were in the non-progression group. This compares with 37 (20.79\%) and 141 (79.21\%), respectively, for the 178 cases of negative HPV infection, and $3(11.54 \%)$ and $23(88.46 \%)$, respectively, for the 26 cases of nononcogenic HPV infection. The relative risk of progression of cytological findings 2 years later in cases of oncogenic HPV infection was higher than in cases of non-oncogenic HPV infection, as well as in cases of negative HPV infection (Table 4). However, the relative risk of progression of cytological findings 2 years later in cases of nononcogenic HPV infection was not higher than in cases of negative HPV infection (Table 4).

\section{Association between single HPV-16 or HPV-52 infection and later cytological findings in Japanese women with NILM or ASC-US} In the 20 cases of single HPV-16 infection (HPV-16 group), 13 (65\%) were classified in the progression group, whereas 7 (35\%) were in the 


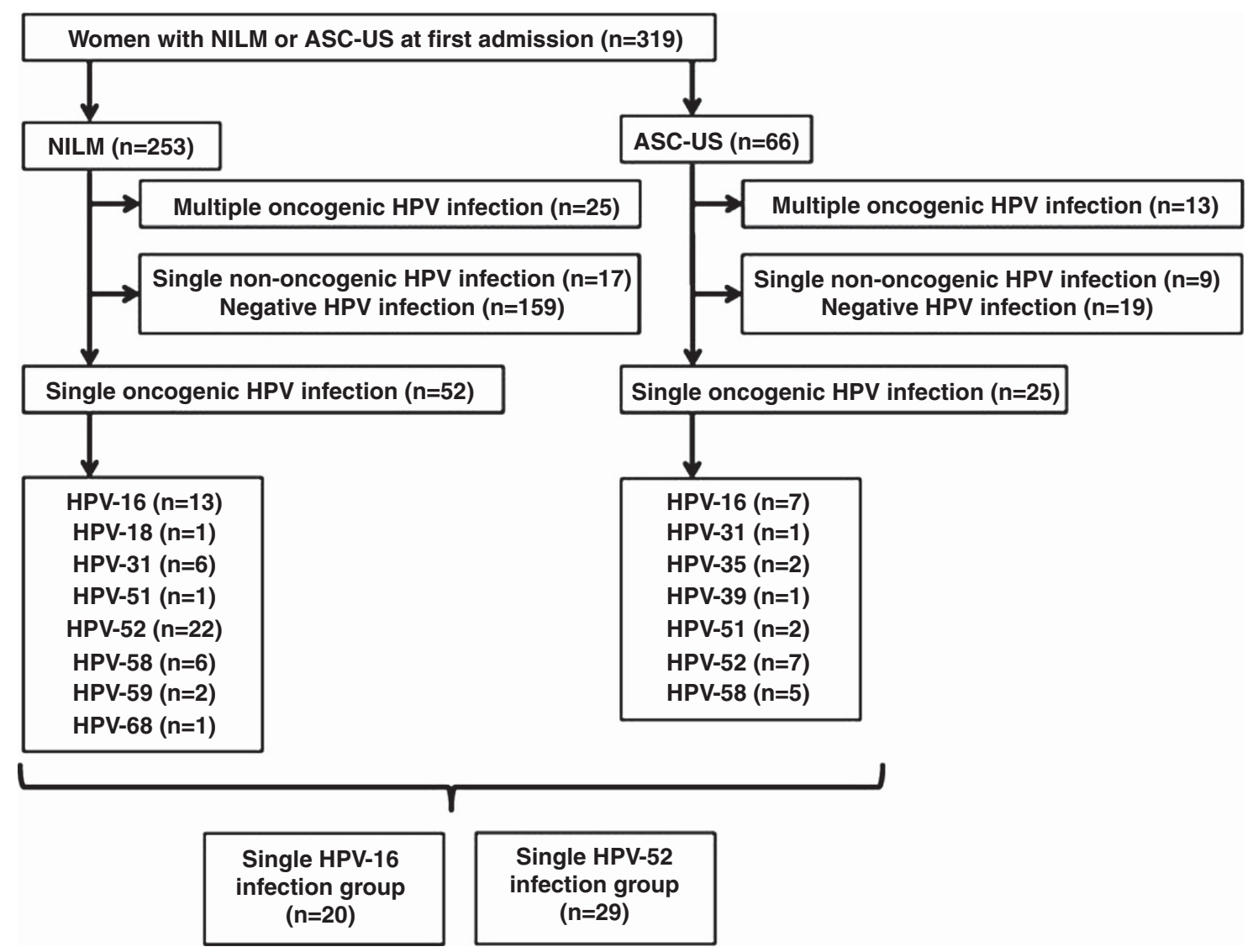

Figure 1 Results of HPV genotyping in women with NILM or ASC-US at first admission. ASC-US, atypical squamous cells of undetermined significance; HPV, human papillomavirus; NILM, negative for intraepithelial lesion and malignancy.

Table 1 Patient background in cases of single oncogenic HPV, non-oncogenic HPV or negative HPV infections

\begin{tabular}{|c|c|c|c|c|c|}
\hline & $A$ & 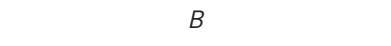 & c & & \\
\hline Age at first sampling (years) ${ }^{a}$ & $42.58(11.78)$ & $40.27(14.18)$ & 41.95 (11.03) & 0.232 & 0.876 \\
\hline Smoking ${ }^{b}$ & $12(15.6 \%)$ & $6(23.1 \%)$ & $21(11.8 \%)$ & 0.384 & 0.408 \\
\hline Use of oral contraceptive ${ }^{b}$ & $3(3.9 \%)$ & $0(0.0 \%)$ & $6(3.4 \%)$ & 0.307 & 0.835 \\
\hline \multicolumn{6}{|l|}{ Parity $^{\mathrm{b}}$} \\
\hline Nulliparous & $24(31.2 \%)$ & $5(19.2 \%)$ & $60(33.7 \%)$ & 0.495 & 0.924 \\
\hline Primiparous & $14(18.2 \%)$ & $6(23.1 \%)$ & $31(17.4 \%)$ & & \\
\hline Multiparous & $39(50.6 \%)$ & $15(57.7 \%)$ & $87(48.9 \%)$ & & \\
\hline Body mass index $\left(\mathrm{kg} \mathrm{m}^{-2}\right)^{\mathrm{a}}$ & $21.32(2.47)$ & $21.17(2.34)$ & $21.27(2.76)$ & 0.963 & 0.727 \\
\hline
\end{tabular}

Abbreviation: HPV, human papillomavirus.

a Mean (s.d.). Student's $t$-test was used to analyze differences between groups. Statistical significance was defined as $P<0.05$.

bNumber of cases (percentage). $\chi^{2}$ test was used to analyze differences between groups. Statistical significance was defined as $P<0.05$.

non-progression group. This compares with $9(31.03 \%)$ and $20(68.97 \%)$, respectively, for the 29 cases of single HPV-52 infection (HPV-52 group). The relative risk of progression of cytological findings 2 years later in cases of HPV-16 infection was higher than in cases of HPV-52 infection (Table 5).

\section{DISCUSSION}

In this study, we investigated the association between common oncogenic HPV infection in a Japanese population and the progression of cytological findings 2 years later in Japanese women with NILM or ASC-US.

Matsumoto et al. ${ }^{10}$ previously reported that in Japanese women with cytological low-grade squamous intraepithelial lesion and histological CIN grade 1-2 lesions, the oncogenic HPV infection group showed a greater tendency to develop CIN3 within 5 years than the non-oncogenic HPV infection group. ${ }^{1}$ Consistent with these results, we confirmed that infection with common oncogenic HPV in Japan poses a higher risk for the progression of cytological findings 
in the uterine cervix 2 years later than non-oncogenic HPV infection or no HPV infection.

As our study cohort was a pre-vaccination population; there is no influence of the HPV vaccine on our observations. Although more spontaneous regression of HPV infection usually occurs among young women, there was no significant difference in age distributions between the progression and the non-progression groups in this study. Therefore, we were able to investigate how the HPV genotype affects later cytological findings in Japanese women with NILM or ASC-US. Our investigation suggests that Japanese women with oncogenic HPV infections should undergo periodic checkups for uterine cervical cancer over the course of 2 years from initial

Table 2 Patient backgrounds in cases of single HPV 16 or single HPV 52 infections

\begin{tabular}{lccc}
\hline & $\begin{array}{c}\text { Single HPV-16 infection } \\
\text { group }(\mathrm{n}=20)\end{array}$ & $\begin{array}{c}\text { Single HPV-52 infection } \\
\text { group ( } \mathrm{n}=29)\end{array}$ & P-value \\
\hline $\begin{array}{l}\text { Age at first sam- } \\
\text { pling (years) }\end{array}$ & $43.60(15.99)$ & $40.83(10.01)$ & 0.768 \\
$\begin{array}{l}\text { Smoking } \\
\text { Use of oral }\end{array}$ & $5(25 \%)$ & $4(13.8 \%)$ & 0.319 \\
$\begin{array}{l}\text { contraceptive } \\
\text { Parity }\end{array}$ & $1(5 \%)$ & $0(0 \%)$ & 0.224 \\
$\quad$ & & & \\
Nulliparous & $7(35 \%)$ & $11(37.9 \%)$ & 0.381 \\
$\begin{array}{l}\text { Primiparous } \\
\text { Multiparous }\end{array}$ & $5(25 \%)$ & $3(10.3 \%)$ & \\
Body mass index & $8(40 \%)$ & $15(51.7 \%)$ & \\
$\quad\left(\mathrm{kg} \mathrm{m}^{-2}\right)^{\mathrm{a}}$ & $21.84(3.27)$ & $21.42(2.15)$ & 0.935 \\
\hline
\end{tabular}

Abbreviation: HPV, human papillomavirus.

a Mean (standard deviation). Student's $t$-test was used to analyze differences between groups.

Statistical significance was defined as $P<0.05$.

bNumber of cases (percentage). $\chi^{2}$ Test was used to analyze differences between groups.

Statistical significance was defined as $P<0.05$.

Table 3 Age distributions between the progression and the nonprogression groups

\begin{tabular}{lccc}
\hline Age (years) & Progression group & Non-progression group & P-value $^{\text {a }}$ \\
\hline $20-29$ & 4 & 35 & \\
$30-39$ & 24 & 72 & 0.06 \\
$\geqslant 40$ & 42 & 104 & \\
Total cases & 70 & 211 &
\end{tabular}

aPearson's $\chi^{2}$ test was used to analyze differences in age distributions between groups. Statistical significance was defined as $P<0.05$. identification of infection. In a previous study by Dufresne et al., ${ }^{11}$ persistent infection of oncogenic HPV was detected in $33(67.6 \%)$ of 34 cases of ASC-US with oncogenic HPV infections, while in the remaining 11 cases (32.4\%), 6 months later oncogenic HPV had disappeared and there was no sign of CIN2/3. In our study, among the 12 single oncogenic HPV-positive women diagnosed with ASCUS at first admission, persistent infection of oncogenic HPV was detected in four $(33.3 \%)$, while oncogenic HPV had disappeared in the remaining eight cases $(66.7 \%) 2$ years later and there was no sign of cytological progression in these eight cases 2 years later. These results confirm that, as time passes, oncogenic HPV can disappear spontaneously in women with ASC-US, and that the negative predictive value of the HPV test may help to reduce the number of unnecessary colposcopies carried out in women with ASC-US. ${ }^{11}$

Khan et al. ${ }^{12}$ reported that among the cases of NILM with HPV-16, the 10-year cumulative rate of CIN3 or cancer was $17 \%$ and the 2 -year cumulative incidence rate of CIN3 or cancer was $9.8 \%$. This is similar to our results, which showed that 3 (15\%) of 20 single HPV16-positive cases of 'NILM or ASC-US' at first admission were diagnosed with ASC-H or HSIL by cytological testing 2 years later (data not shown). Recently, the ATHENA (Addressing the Need for Advanced HPV Diagnostics) study reported that, among cases of NILM or ASC-US, the estimated absolute risk of CIN2 or worse in cases of HPV-16 and/or HPV-18 was higher than in cases of other oncogenic HPV. ${ }^{13-15}$ Our result is consistent with these studies that showed HPV-16 is associated with a higher risk of cervical cancer onset than oncogenic HPV genotypes in other regions. ${ }^{16}$ We clarified that a single HPV-16 infection of women with 'NILM or ASC-US'

Table 5 Association between common oncogenic HPV genotype in Japan and progression of cytological findings 2 years later

\begin{tabular}{lccccc}
\hline & \multicolumn{2}{c}{ Results of HPV test } & & \\
\cline { 2 - 3 } & $\begin{array}{c}\text { A } \\
\text { Single HPV-16 } \\
\text { infection }\end{array}$ & $\begin{array}{c}\text { Single HPV-52 } \\
\text { infection }\end{array}$ & Total & cases & $\begin{array}{c}\text { Relative risk (95\% } \\
\text { confidence interval) } \\
\text { A vs B }\end{array}$ \\
\hline $\begin{array}{l}\text { Progression } \\
\text { group }\end{array}$ & 13 & 9 & 22 & 2.094 (1.005-3.935) \\
$\begin{array}{l}\text { Non-progres- } \\
\text { sion group } \\
\text { Total cases }\end{array}$ & 7 & 20 & 27 & \\
\hline
\end{tabular}

Abbreviation: HPV, human papillomavirus.

Table 4 Association between HPV infection and progression of cytological findings 2 years later

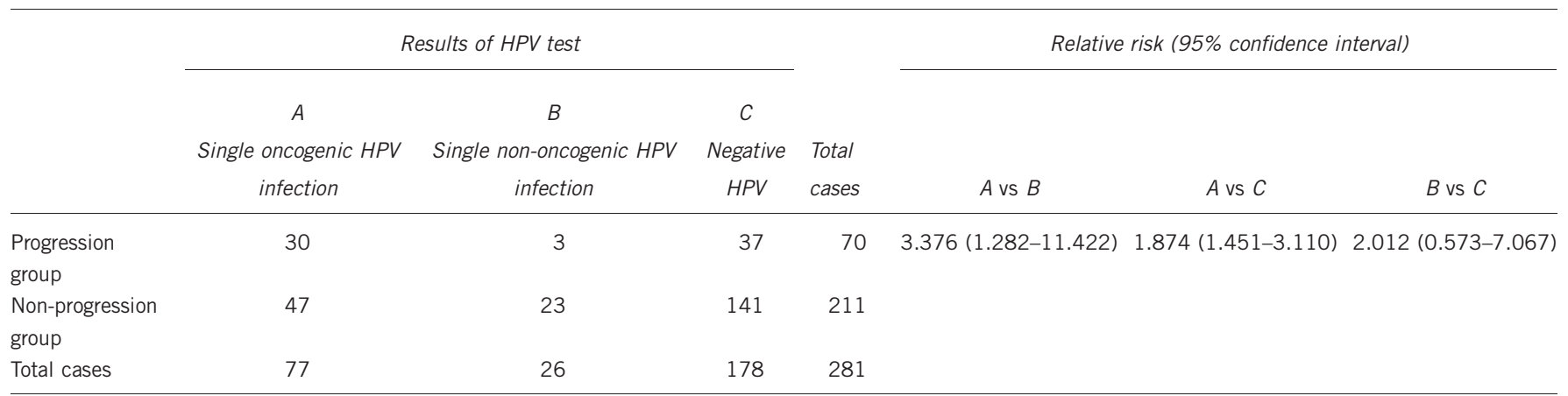

Abbreviation: HPV, human papillomavirus. 
cytology is associated with a higher risk of progression of cytological findings 2 years later compared with a single HPV-52 infection, supporting the recommendation of colposcopy for HPV-16 genotyping of women with NILM or ASC-US cytology. ${ }^{6}$

According to the Cochrane Database of Systematic Reviews, HPVtriage with hybrid capture 2 is recommended to triage women with ASC-US because of its greater accuracy (significantly higher sensitivity, and similar specificity) compared with repeat cytology. ${ }^{17}$ Regarding uterine cervical cancer in Japanese women, the genetics of HPV infection (oncogenic HPV genotype and oncogenic HPV viral loads) is not sufficient to cause CIN3/cervical cancer, and host genetic factors are also likely to contribute to cervical cancer pathogenesis. $^{2,4,5,8,18-22}$ Further study focused on cancer genetics in both the HPV virus and host may enable the development of a comprehensive system of early detection and prevention of uterine cervical cancer.

In conclusion, for the first time, we confirmed that common oncogenic HPV infection is associated with a higher risk of progression of cytological findings 2 years later in Japanese women with NILM or ASC-US; of these infections, HPV-16 was found to have a higher risk of progressive cytological findings than HPV-52. Therefore, the initial HPV-DNA genotype may be a potential predictive marker of later progression of cytological findings in the uterine cervix in Japanese women with NILM or ASC-US.

\section{CONFLICT OF INTEREST}

The authors declare no conflict of interest.

\section{ACKNOWLEDGEMENTS}

This work was supported by the Japan Society for the Promotion of Science KAKENHI, grant numbers 23592406 and 24791712. We thank Makoto Murakami, Masaki Fuse, Tetsuro Samejima, Akira Fujishita, Daisuke Nakayama, Kohei Kotera and Yasuko Noguchi for their assistance in this study.

1 Muñoz, N., Bosch, F. X., de Sanjosé, S., Herrero, R., Castellsagué, X., Shah, K. V. et al. The International Agency for Research on Cancer Multicenter Cervical Cancer Study Group. Epidemiologic classification of human papillomavirus types associated with cervical cancer. New Engl. J. Med. 348, 518-527 (2003).

2 Yamasaki, K., Miura, K., Shimada, T., Ikemoto, R., Miura, S., Murakami, M. et al. Pre-vaccination epidemiology of human papillomavirus infections in Japanese women with abnormal cytology. J. Obstet. Gynaecol. Res. 37, 1666-1670 (2011).

3 Yamasaki, K., Miura, K., Shimada, T., Miura, S., Abe, S., Murakami, M. et al. Epidemiology of human papillomavirus genotypes in pregnant Japanese women. J. Hum. Genet. 56, 313-315 (2011).

4 Inoue, M., Okamura, M., Hashimoto, S., Tango, M. \& Ukita, T. Adoption of HPV testing as an adjunct to conventional cytology in cervical cancer screening in Japan. Int. J. Gynaecol. Obstet. 111, 110-114 (2010).

5 Smith, J. S., Lindsay, L., Hoots, B., Keys, J., Franceschi, S., Winer, R. et al. Human papillomavirus type distribution in invasive cervical cancer and high-grade cervical lesions: a meta-analysis update. Int. J. Cancer 121, 621-632 (2007).
6 Saslow, D., Solomon, D., Lawson, H. W., Killackey, M., Kulasingam, S. L., Cain, J. et al. American Cancer Society; American Society for Colposcopy and Cervical Pathology; American Society for Clinical Pathology. American Cancer Society, American Society for Colposcopy and Cervical Pathology, and American Society for Clinical Pathology screening guidelines for the prevention and early detection of cervical cancer. Am. J. Clin. Pathol. 137, 516-542 (2012).

7 Gravitt, P. E., Peyton, C. L., Alessi, T. Q., Wheeler, C. M., Coutlée, F., Hildesheim, A. et al. Improved amplification of genital human papillomaviruses. J. Clin. Microbiol. 38, 357-361 (2000).

8 Asato, T., Maehama, T., Nagai, Y., Kanazawa, K., Uezato, H. \& Kariya, K. A large casecontrol study of cervical cancer risk associated with human papillomavirus infection in Japan, by nucleotide sequencing-based genotyping. J. Infect. Dis. 189, 1829-1832 (2004).

9 Walboomers, J. M., Jacobs, M. V., Manos, M. M., Bosch, F. X., Kummer, J. A., Shah, K. V. et al. Human papillomavirus is a necessary cause of invasive cervical cancer worldwide. J. Pathol. 189, 12-19 (1999).

10 Matsumoto, K., Oki, A., Furuta, R., Maeda, H., Yasugi, T., Takatsuka, N. et al. Japan HPV and Cervical Cancer Study Group. Predicting the progression of cervical precursor lesions by human papillomavirus genotyping: a prospective cohort study. Int. J. Cancer 128, 2898-2910 (2011).

11 Dufresne, S., Sauthier, P., Mayrand, M. H., Petignat, P., Provencher, D., Drouin, P. et al. Human papillomavirus (HPV) DNA triage of women with atypical squamous cells of undetermined significance with amplicor HPV and hybrid capture 2 assays for detection of high-grade lesions of the uterine cervix. J. Clin. Microbiol. 49, 48-53 (2011).

12 Khan, M. J., Castle, P. E., Lorincz, A. T., Wacholder, S., Sherman, M., Scott, D. R. et al. The elevated 10-year risk of cervical precancer and cancer in women with human papillomavirus (HPV) type 16 or 18 and the possible utility of type-specific HPV testing in clinical practice. J. Natl Cancer Inst. 97, 1072-1079 (2005).

13 Stoler, M. H., Wright, T. C. Jr, Sharma, A., Apple, R., Gutekunst, K. \& Wright, T. L. ATHENA (Addressing the Need for Advanced HPV Diagnostics) HPV Study Group. High-risk human papillomavirus testing in women with ASC-US cytology: results from the ATHENA HPV study. Am. J. Clin. Pathol. 135, 468-475 (2011).

14 Wright, T. C. Jr, Stoler, M. H., Sharma, A., Zhang, G., Behrens, C. \& Wright, T. L. ATHENA (Addressing the Need for Advanced HPV Diagnostics) Study Group. Evaluation of HPV-16 and HPV-18 genotyping for the triage of women with high-risk HPV + cytology-negative results. Am. J. Clin. Pathol. 136, 578-586 (2011).

15 Stoler, M. H., Wright, T. C. Jr, Sharma, A., Zhang, G., Apple, R., Wright, T. L. et al. ATHENA (Addressing the Need for Advanced HPV Diagnostics) Study Group. The interplay of age stratification and HPV testing on the predictive value of ASC-US cytology. Results from the ATHENA HPV study. Am. J. Clin. Pathol. 137, 295-303 (2012).

16 Bouvard, V., Baan, R., Straif, K., Grosse, Y., Secretan, B., El Ghissassi, F. et al. A review of human carcinogens-part B: biological agents. Lancet Oncol. 10, 321-322 (2009).

17 Arbyn, M., Roelens, J., Simoens, C., Buntinx, F., Paraskevaidis, E., Martin-Hirsch, P. P. et al. Human papillomavirus testing versus repeat cytology for triage of minor cytological cervical lesions. Cochrane Database Syst. Rev. 3, CD008054 (2013).

18 Magnusson, P. K., Lichtenstein, P. \& Gyllensten, U. B. Heritability of cervical tumours. Int. J. Cancer 88, 698-701 (2000).

19 Yoshida, T., Sano, T., Kanuma, T., Owada, N., Sakurai, S., Fukuda, T. et al. Quantitative real-time polymerase chain reaction analysis of the type distribution, viral load, and physical status of human papillomavirus in liquid-based cytology samples from cervical lesions. Int. J. Gynecol. Cancer 18, 121-127 (2008).

20 Hamaguchi, Y., Miura, K., Abe, S., Kinoshita, A., Miura, S., Yamasaki, Y. et al. Initial viral load in cases of single human papillomavirus 16 or 52 persistent infection is associated with progression of later cytopathological findings in the uterine cervix. J. Med. Virol. 85, 2093-2100 (2013).

21 Matsumoto, K., Maeda, H., Oki, A., Takatsuka, N., Yasugi, T., Furuta, R. et al. HLA class II DRB1*1302 allele protects against progression to cervical intraepithelial neoplasia grade 3: a multicenter prospective cohort study. Int. J. Gynecol. Cancer 22, 471-478 (2012).

22 Abe, S., Miura, K., Kinoshita, A., Mishima, H., Miura, S., Yamasaki, K. et al. Copy number variation of the antimicrobial-gene, defensin beta 4 , is associated with susceptibility to cervical cancer. J. Hum. Genet. 58, 250-253 (2013). 